\title{
COLOR Y QUALIA. NI REPRESENTACIONISMO NI FENOMENISMO
}

ANDONI IBARRA

Unidad de Estudios sobre la Ciencia y la Tecnología

CSIC-Universidad del País Vasco

ylpibuna@sf.ehu.es

EKAI TXAPARTEGI

Departamento de Lógica y Filosofía de la Ciencia

Universidad del País Vasco

ylbtxzue@sf.ehu.es

RESUMEN: El debate entre representacionistas y fenomenistas acerca del realismo de los qualia parece no avanzar. Este artículo defiende una solución que no es ni representacionista ni fenomenista. En contra de los representacionistas mantenemos que no todo contenido perceptual es reducible a su contenido representacional. En contra de los fenomenistas sostenemos que todo contenido perceptual es contenido intencional. Negamos así la existencia de los qualia, de aquellos, al menos, caracterizados de manera más estándar. Finalmente, mostramos que nuestra propuesta — situada entre el representacionismo y el fenomenismo - no ha sido explorada, porque se ha asumido, erróneamente, que todo contenido no representacional debe ser contenido no intencional.

PALABRAS CLAVE: qualia, color, funcionalismo, contenido representacional

SUMMARY: The debate between representationalists and phenomenalists on the reality of qualia has stagnated. The present article argues for a solution that is neither representationalist nor phenomenalist. Unlike the representationalists, we hold that not all perceptual content is reducible to its representational content. Against the phenomenalists, we claim that all perceptual content is intentional content. We therefore discard the existence of qualia, at least in their standard guise. Finally, we show that our intermediate proposal has not been explored because until now all non-representational content has been erroneously understood to be non-intentional content.

KEY WORDS: qualia, color, functionalism, representational content

\section{Introducción}

Dos debates se han simultaneado tradicionalmente: el primero concierne a la existencia de los qualia, y el segundo a la naturaleza constitucional de los colores. En la tradición, en efecto, 
para decidir acerca de la existencia de los qualia parecía imprescindible analizar qué son los colores. La filosofía de la mente ha vinculado el análisis de la existencia o no de qualia en el mundo sobre todo al estudio de los hechos cromáticos y las hipótesis cromáticas según los distintos mundos concebibles. Sin embargo, a pesar de los esfuerzos realizados en esa dirección, el debate entre fenomenistas y representacionistas no parece haber conducido a resultados estables. Por eso, en este artículo se contempla la posibilidad de ofrecer un punto de partida alternativo al situado en el origen del debate tradicional. Quizá ya no quedan más mundos cromáticamente posibles por examinar. Quizá ya no quedan más hechos cromáticos pertinentes por descubrir. Así las cosas, podríamos entonces plantearnos la pregunta "¿qué es lo que puede remover el debate en torno a la realidad o no de los qualia?" Una respuesta sería afirmar que todo el debate ha sido fruto de una confusión terminológica. Aquí la tomaremos como nuestro punto de partida. Pero, ¿cuál ha sido exactamente la confusión? Y, ¿por qué ha perdurado tanto? Estas preguntas determinan el marco temático del presente artículo.

Con frecuencia, la historiografía se expresa como una narración de guerras o conflictos sucesivos. Incluso la pacífica historia de la filosofía se inscribe en este registro. Una de las guerras más relatadas en el campo de la filosofía de la mente es la guerra de los qualia. Según el relato oficial, en un bando se sitúan los fenomenistas enarbolando el lema "iVivan los qualia!"; desde el otro bando, los representacionistas responden "¡Abajo con ellos!" Se ha discutido mucho en y sobre esta guerra: quién y por qué ha dicho qué; cómo y cuándo se le ha respondido, etc. Pero todavía queda por preguntar "dónde" se dirime la batalla y cuáles son las implicaciones que tiene la determinación del "lugar" elegido para desarrollar la contienda. Nuestra idea es que el campo de batalla donde se sitúa habitualmente la actividad polémica determina de manera esencial el acontecer de la guerra de los qualia. En particular, creemos que el lugar elegido como teatro de operaciones, esto es, el de la metafísica de los colores, no permite dirimir satisfactoriamente esa guerra. Las respectivas intuiciones, tanto sobre los colores como sobre 
las experiencias cromáticas, son instrumentalizadas con el único propósito de proveer el material con el cual ambos bandos abastecen continuamente su guerra. A nuestro juicio, sin embargo, la metafísica de los colores no es el ámbito adecuado para decidir sobre la realidad de los qualia. Ponemos en duda, en concreto, la legitimidad de la utilización instrumental de los colores a favor o en contra de la existencia de los qualia. Tanto para comprender la naturaleza de los colores como para destrabar el enconado debate sobre el realismo acerca de los qualia, conviene analizar los colores partiendo de un interés focal en los colores mismos y no de intereses previos asociados al mantenimiento de determinadas posturas en otros problemas genuinos de la filosofía de la mente.

Enunciado brevemente, el estado de la cuestión en el relato de la guerra de los qualia es el siguiente. Los representacionistas mantienen que las experiencias cromáticas siempre son intencionales, es decir, siempre son sobre algo. Los colores se perciben y la introspección no desempeña ningún papel. De hecho, en contra de lo sostenido por los viejos filósofos, las experiencias cromáticas de cada sujeto normal son identificables de manera objetiva y sus referencias son definibles ostensivamente. Más aún, hoy disponemos de los medios científicos necesarios para detectar las diferencias entre las experiencias cromáticas de los distintos individuos. Los fenomenistas, por el contrario, apoyándose en las ciencias cognitivas, se comprometen con la idea de que las experiencias perceptuales no son meramente operacionales. Según ellos, las experiencias cromáticas tienen siempre un componente cualitativo no reducible a su contenido representacional. Además, prueban este hecho mediante experimentos mentales que tienen como fin mostrar los límites del programa reductivo funcionalista. En este estado de cosas, tanto los fenomenistas como los representacionistas recurren a los colores. Todos ellos piensan que los colores aportarán la prueba definitiva necesaria para imponerse al rival de manera concluyente.

Para alcanzar los objetivos de este artículo seguiremos la siguiente estrategia. En las dos primeras secciones describimos 
el modo como los fenomenistas defienden la existencia de los qualia. Esta descripción es pertinente porque mostrará el supuesto fundamental asumido tanto por los fenomenistas como por los representacionistas. Según nuestra interpretación, ambos bandos asumen que todo aquel contenido perceptual que no sea representacional debe ser contenido no intencional. Las secciones 3 y 4 tienen como objetivo combatir ese supuesto compartido. Así, en la tercera sección mostramos que considerar las experiencias cromáticas como no intencionales carece de fundamento "fenomenológico". En la cuarta sección establecemos la distinción analítica entre el carácter cualitativo del campo visual (el aspecto fenoménico) y su contenido atributivo o representacional. Nuestro propósito es mostrar la necesidad de distinguir entre las condiciones que posibilitan la definición ostensiva de los colores y las que posibilitan el uso atributivo de dichos colores ostensivamente definidos porque, si se acepta dicha distinción, se debe aceptar también que los colores son intencionales, incluso aunque carezcan de contenido representacional, funcional o cognitivo. De esta manera se mina el supuesto compartido por los fenomenistas y los representacionistas y se abre la posibilidad de explorar una tercera postura intermedia que, por un lado, niegue la existencia de los qualia pero que, por otro, no requiera asumir que todo contenido perceptual es contenido representacional.

\section{Los qualia no se "filtran"}

Los qualia se pueden caracterizar de manera que resulta difícil negar su existencia: por ejemplo, como contenidos cualitativos o en primera persona de las experiencias perceptuales. Así caracterizados, intuitivamente aceptaríamos que existen; sin embargo, en esa misma caracterización, los qualia ya están determinando un límite para la ciencia actual, en la medida en que se sustraen al estudio científico. Ambas características configuran a los qualia como espectros, fantasmas, que aparecen pero que carecen de cualquier realidad. Así pues, por un lado, disponemos de argumentos "fenoménicos" para pensar que existen pero, por otro lado, tenemos razones "científicas" para pensar 
que no existen. Ned Block (1996, pp. 20 y 30) distinguió dos bandos y bautizó como fenomenistas a aquellos que creen en la existencia de los qualia y como representacionistas a aquellos que la niegan. ¿Qué es lo que puede inclinar la balanza hacia uno u otro bando?

A esta pregunta, los fenomenistas responden que la prueba de la existencia de los qualia está dada por el fracaso del programa funcionalista de reducción de todo contenido de la experiencia perceptual (Block 1999, p. 487). Los fenomenistas tratan de mostrar que el funcionalismo - el programa reductivo con mayor proyección en la filosofía de la mente - no puede reducir completamente las experiencias perceptuales (en particular, las experiencias cromáticas). La maniobra más convincente para demostrar la existencia de los qualia es, en este sentido, la que llamaremos estrategia del filtro. ${ }^{1}$ Esta estrategia establece un paralelismo entre lo que es funcionalmente detectable y el contenido representacional de las experiencias cromáticas de manera que, como se asume que todo contenido representacional es funcionalmente describible, aquello que no lo sea constituye una prueba de la existencia de los qualia. Concretamente, los fenomenistas tratan de mostrar que en la experiencia cromática hay cierto contenido no representacional, esto es, no reducible a su descripción funcional. Los qualia no se "filtran" por decirlo así y, por tanto, aquello que no es reducible, esto es, aquello que queda retenido en el filtro, son, según la interpretación fenomenista, los qualia.

Para mostrar cómo ha funcionado esta estrategia del filtro describiremos dos de los ejemplos más característicos: la tierra invertida y el color mental. El ejemplo de la tierra invertida plantea una situación imaginaria -empíricamente imposible-, que ha sido analizada sobre todo por Harman (1996) y Block

1 A ella se suscribirían los argumentos más conocidos: el espectro invertido (Shoemaker 1982), el murciélago de Nagel (1974), la supercientífica Mary (Jackson 1986), el de los absent qualia y el argumento del vacío explicativo. Y también otros menos conocidos: Fred, que puede percibir un nuevo color (Jackson 1982); Eliza, que está encerrada en una habitación donde los colores se relacionan con las superficies de una manera espontánea y arbitraria (Block 1996), etcétera. 
(1996). El segundo ejemplo, por el contrario, es una interpretación realizada por Macpherson (2003) de un hecho cromático real (aunque su reconocimiento no está exento de cierta polémica) descubierto por Crane y Piantanida (1983): el color mental.

El hipotético experimento de la tierra invertida recrea un viaje imposible a una tierra en la que los colores están simétricamente invertidos respecto de los de nuestro planeta. El viaje lo realiza un astronauta a quien durante el trayecto se le ha invertido el espectro cromático - así como el color del cuerpo-. Intuitivamente podemos postular que el astronauta en esa tierra y nosotros en nuestra tierra tenemos los mismos qualia a pesar de que, comparativamente, esos qualia tienen distinto contenido representacional en uno y otro caso. Es decir, tanto el astronauta como nosotros percibimos, por ejemplo, que el cielo es azul pero con una diferencia fundamental: siempre según la interpretación fenomenista, la percepción del astronauta no es verdadera porque, por hipótesis, en la tierra invertida el cielo tiene color amarillo. Según esta hipótesis, hay algo funcionalmente indetectable, ya que los dos tipos de sujetos son funcionalmente equivalentes, pero que hace que - si compartimos las intuiciones fenomenistas - las percepciones del astronauta no puedan considerarse verdaderas. ${ }^{2}$ Ese algo serían los qualia.

Nuestro segundo ejemplo, el del color mental, es resultado de un experimento empírico reproducible. Este experimento de

2 La objeción representacionista de Harman ataca esa intuición fenomenista. Si el astronauta es funcionalmente indistinguible respecto de sus nuevos vecinos invertidos, no hay razón alguna para mantener que, a pesar de ello, las percepciones cromáticas del astronauta no sean verdaderas. Por su parte, Block acepta que, transcurrido cierto tiempo, las percepciones cromáticas del astronauta en la tierra invertida serán verdaderas. Pero, en ese caso, el astronauta sufre un cambio en el contenido representacional de las experiencias cromáticas sin que varíen los qualia. Por lo tanto, el representacionismo tiene sólo dos opciones, ambas poco deseables: o acepta que el contenido representacional no varía en el tiempo y entonces debe asumir la posibilidad de sujetos funcionalmente equivalentes pero con contenido representacional diferente o, por el contrario, acepta que el contenido representacional varía con el tiempo y entonces debe asumir que hay algo que no varía, y que es irreducible al contenido representacional. Según Block, Harman no puede aceptar ninguna de las dos opciones. 
laboratorio se basa en un conocido mecanismo psicológico de compleción, según el cual el cerebro "completa" por su cuenta aquello que percibe cuando no le llega la información retinal de un determinado campo. En este caso, en concreto, el cerebro debe "completar" una determinada zona del campo visual pero con la peculiaridad de que la información cromática de que dispone es contradictoria. El resultado del experimento es que, para completar esa área del campo visual, el cerebro utiliza un color que, según la fisiología del sistema perceptual, no sería alcanzable según el procedimiento habitual, es decir, por medio de la estimulación retinal. Por lo tanto, si se acepta el resultado de dicho experimento, el sujeto experimenta un color típicamente mental; es decir, un color que no se puede percibir mediante la estimulación del ojo, pero que nuestra mente sí puede proyectar. Si aceptamos que ese color mental es un color, debemos aceptar la existencia de los qualia, porque tales experiencias cromáticas - al menos en nuestra configuración actual- carecen de contenido representacional. Así, los fenomenistas concluyen que los colores mentales son qualia.

Como se ve, la estrategia de los fenomenistas consiste en preparar las experiencias cromáticas para su filtrado, presentando contraejemplos a la tesis representacionista según la cual todo contenido perceptual es — reducible a su - contenido representacional. Los fenomenistas tratan de mostrar que la experiencia perceptual no es transparente, que no todo se filtra. Así, el ejemplo de la tierra invertida muestra que a veces algo pasa por el filtro y algo distinto se queda, y el ejemplo del color mental muestra que a veces todo su contenido queda sin filtrarse.

Ahora bien, ¿es suficiente esta plural estrategia del filtro para mostrar la existencia de los qualia? Parece que no. Si aceptamos los resultados mencionados, lo único que esa estrategia muestra por sí misma es la corrección del siguiente condicional: si lo que no se filtra son qualia, los qualia existen. Ahora bien, ¿cómo justifican los fenomenistas que lo que no se filtra sean qualia? Lo que queda en el aire es el supuesto previo, esto es, la idea de que todo contenido que no sea representacional debe ser considerado un quale. ¿Por qué se asume ese supuesto? La 
respuesta se sitúa, a nuestro juicio, en otro trasfondo, a saber, en el del debate tradicional entre los subjetivistas y los objetivistas acerca de la naturaleza constitucional del color (ello explica también por qué las experiencias perceptuales que se someten a la prueba del filtrado son, habitualmente, las experiencias cromáticas). Si se acepta que las dos opciones posibles sobre esa naturaleza del color son la objetivista y la subjetivista, entonces la manera más natural de interpretar el fracaso del funcionalismo es asumiendo el subjetivismo; es decir, aceptando que aquello que no se ha filtrado son datos sensoriales proyectados o, dicho de otra manera, qualia. De hecho, los fenomenistas presentan sus resultados favorables a la existencia de los qualia como resultados que favorecen también la posición subjetivista - o su variante disposicionalista - acerca del color.

\section{Se proyectan}

Acabamos de indicar que el fenomenismo supone que aquello que no se ha filtrado son los qualia; pero, ¿qué son los qualia? Su caracterización negativa, en cuanto contenidos no representacionales de la experiencia perceptual, no constituye una definición suficientemente satisfactoria, porque afirmar que los qualia no son contenidos representacionales es tanto como decir que son entidades mentales en un sentido funcionalmente irreducible, con lo que estaríamos abocados a un círculo vicioso. Por otra parte, la caracterización intuitiva de los qualia como contenidos cualitativos o en primera persona, "cómo es ser o tener" (what it's like), tampoco resulta satisfactoria porque no es suficientemente precisa. Parece requerirse entonces una definición teórica, no meramente ostensiva, de los qualia. En concreto necesitaríamos una definición precisa que acabara con la ambigüedad asociada a las múltiples interpretaciones de eso que señala la definición ostensiva: ${ }^{3}$ en suma, necesitaríamos definir positivamente los qualia de manera más precisa.

${ }^{3}$ Esta variedad interpretativa se refleja en los múltiples modos de referirse a ese eso, que los fenomenistas mantienen que no se filtra: el "experimentar" (experiencing), el "cómo es ser o tener" (what it's like), contenido cualitativo, contenido sensacional, propiedad cualitativa, etcétera. 
Aceptaremos como punto de partida la caracterización de los qualia - proveniente de la tradición de los datos sensorialescomo contenidos no intencionales de la experiencia proyectados al exterior.

Los qualia son contenidos no intencionales de las experiencias perceptuales. Formalizamos la intencionalidad como ' $\rightarrow$ ', símbolo que vincula dos ámbitos en una relación todavía sin especificar. Esta relación no es ni de inferencia, ni de consecuencia, ni estrictamente predicativa. "Está soltero, luego no está casado", "llueve, luego la calle se moja" o "el jardín es verde" no son relaciones intencionales. Antes bien, en línea con Brentano, la intencionalidad debe entenderse como cierta propiedad que tienen algunos estados y sucesos mentales, según la cual están dirigidos a o son sobre o de objetos y estados de cosas en el mundo. ' $\rightarrow$ ' simboliza, por lo tanto, la relación intencional que surge cuando algo es "de" o "sobre" otro algo (para algún sujeto). En este sentido, los ejemplos paradigmáticos son los llamados contextos intencionales en los que un sujeto cree, piensa, sugiere, desea, percibe, ..., cualquier cosa. Nuestras actitudes proposicionales, así como nuestras percepciones, son sobre cualquier cosa (la última jugada de Ronaldo, el Guernica de Picasso, etc.); por eso consideramos que nuestras actitudes proposicionales son intencionales, porque normalmente tienen contenido representacional:

$$
\mathrm{Sf}(\mathrm{x}) \text { donde } \mathrm{x} \rightarrow x .
$$

Esto es, un sujeto, $\mathrm{S}$, tiene cierta actitud proposicional, $\mathrm{f}(\mathrm{x})$, cuyo contenido representacional, $\mathrm{x}$, se refiere a algo que normalmente no es intencional, $x$. Es decir, el predicado "Haber oído que el jorobado de París tiene un metro de altura" es intencional, en el sentido de que se refiere a algo que alguien ha oído sobre un determinado individuo que se encuentra en París.

Regresemos entonces a la estrategia del filtro. Cuando los fenomenistas afirman que lo no filtrado son los qualia, lo que quieren decir es que hay ciertos contextos intencionales cuyo contenido no es intencional, 
S-percibe- $(q)$,

tal que $q$ es un quale - por ejemplo, rojo- En el contexto intencional "S-percibe- $(q)$ ", aquello que debería cumplir el papel del contenido representacional, $q$, no es intencional, esto es, no se refiere a otra cosa, q-y, por lo tanto, tampoco se considera contenido representacional- Por eso, según los fenomenistas, $q$ tampoco es funcionalmente reducible; de ahí que no se filtre. No es de extrañar, entonces, que el escepticismo o el idealismo hayan surgido como consecuencia del realismo acerca de los qualia: porque, efectivamente, es difícil mostrar cómo una entidad no intencional, que supuestamente media en todo acto perceptual, puede permitir la percepción directa de la realidad. Por eso, para que sea posible el conocimiento del mundo exterior, el representacionismo debe combinarse con el antirrealismo acerca de los qualia. ${ }^{4}$

Cabe preguntarse entonces si el fenomenista ha ofrecido algún argumento adicional para pensar que todo lo no filtrado, todo aquello que no es contenido representacional, es contenido no intencional. La respuesta es afirmativa. De hecho, normalmente la estrategia del filtro se combina con el argumento de los intencionales inexistentes (las imágenes residuales, las alucinaciones, los fenómenos fosfeno, los colores mentales, etc.), precisamente para mostrar que aquello que no se filtra son, justamente, contenidos no intencionales de la experiencia. Esta serie de fenómenos cromáticos mostraría que, al menos en esos casos, los colores no son intencionales, en el sentido de que no

${ }^{4}$ Más aún, ciertas teorías fenomenistas de la intencionalidad consideran que el contenido informacional "indirecto" de las experiencias cromáticas (como "ser causado por" o "estar en la situación neurofisiológica tal", etc.) puede ser concebido como contenido representacional de dichas experiencias. Sin embargo, esta postura fuerza en exceso lo que queremos decir con "contenido representacional" en el presente contexto. La intencionalidad es una propiedad de los estados y sucesos mentales y, si se flexibiliza excesivamente en la dirección de esa postura, todo suceso no mental tendría su propio contenido representacional. Lo que Sherlock Holmes busca en el escenario del crimen son pruebas, no contenidos representacionales. Un cuchillo manchado de sangre puede contener huellas del asesino pero no contenidos representacionales. 
se refieren a ninguna región del mundo físico. La fuerza del argumento reside en la fenomenología de los casos perceptuales mencionados. En contra de la tesis de la intencionalidad, ese tipo de colores no se localizan en el exterior, no representan ninguna localización exterior, y, por lo tanto, se perciben sin que correspondan a algo exterior, esto es, sin que sean sobre algo (en realidad nunca existió el puñal que Macbeth creyó percibir, era una alucinación). En esos casos, según la interpretación fenomenista, los qualia se perciben como lo que son, sin que la mente los haya proyectado todavía a ninguna superficie. Los intencionales inexistentes constituyen, pues, según los fenomenistas, la prueba de que, en un estadio preperceptual, los qualia son entidades no intencionales que, en un estadio posterior, la mente proyecta a la realidad exterior. Estos fenómenos cromáticos muestran que la proyección, que no es sino un modo metafórico de referirnos a la intencionalidad, es algo que los qualia adquieren en un estadio posterior y, por lo tanto, no es constitutiva de su identidad.

En definitiva, los fenomenistas prueban la existencia de los qualia - los contenidos no intencionales de la experiencia perceptual-, combinando la estrategia del filtro con una interpretación no intencional de los intencionales inexistentes. Sin embargo, desafortunadamente para los fenomenistas, todo ese esfuerzo por mostrar los límites del funcionalismo y la no intencionalidad de nuestras experiencias cromáticas más básicas no ha servido para cerrar el debate sobre la existencia de los qualia. Todo lo contrario: los representacionistas tienen preparada la respuesta para cada aspecto de la discusión — los supuestos límites del funcionalismo o la caracterización no intencional de las experiencias cromáticas-.

\section{Referirse sin representar}

Hasta ahora se ha presentado la estrategia utilizada por los fenomenistas para defender su realismo acerca de los qualia. Esta estrategia consiste, básicamente, en combinar la estrategia del filtro con una concepción subjetivista de los colores. Hemos soslayado, por otro lado, la presentación y la evaluación de la con- 
trarréplica representacionista, porque no es nuestra intención volver a analizar los argumentos particulares a favor o en contra de la existencia de los qualia - argumentos suficientemente conocidos_-, sino calibrar la propia legitimidad de los términos en los que se ha desarrollado el debate entre fenomenistas y representacionistas. Como ya adelantamos, lo que pretendemos es cuestionar la legitimidad del presupuesto del debate y, si nuestra argumentación es razonable, ello planteará un serio problema tanto a los fenomenistas como a los representacionistas. Consideremos la siguiente estipulación:

(A) Existen ciertas circunstancias en las cuales las experiencias cromáticas se refieren a cierta localización espaciotemporal, sin que por ello representen cromáticamente aquello a lo que se refieren.

A nuestro entender, ni los fenomenistas ni los representacionistas pueden asumir (A). Al menos esto no es posible en el formato en el que se desarrolla el debate entre ellos. En contra de los representacionistas, (A) mantiene que puede haber circunstancias en las que las experiencias cromáticas carezcan de contenido representacional. En contra de los fenomenistas, en cambio, (A) mantiene que lo no filtrado no son qualia, porque son intencionales incluso en los casos que carecen de contenido representacional. De hecho, se dice que las experiencias cromáticas se refieren a cierta localización espaciotemporal (superficie, volumen, fuente de luz, etc.). Es decir, si se acepta (A), se está afirmando que en las experiencias cromáticas hay algo - que en adelante llamaremos "aspecto fenoménico" - que es intencional, pero que no necesariamente es representacional. De manera más concreta, lo que se quiere decir es que, en ciertos contextos, el aspecto fenoménico no es representacional; sin embargo, no por eso hay que suponer que en ellos el aspecto fenoménico no sea intencional (ni siquiera en los contextos formulados por los casos de los intencionales inexistentes). Esos contextos plantean la posibilidad de una postura distinta del "fenomenismo" — que es realista respecto de los qualia - y del "representacionismo" —el cual considera que todo contenido 
perceptual es representacional-: ni todo contenido perceptual es representacional —ni, por lo tanto, reducible a contenidos más básicos-, ni el contenido no representacional es necesariamente no intencional.

Para justificar (A) utilizaremos dos estrategias. La primera, que se presenta en la siguiente sección, parte de una concepción representacionista de la naturaleza del color y justifica (A) como parte de esa concepción general acerca de los colores. La segunda estrategia, que se presenta a continuación, es más sencilla. Simplemente exige otra prueba, más sólida, a los fenomenistas. Esta segunda estrategia consiste en no dar crédito al argumento de los intencionales inexistentes como prueba antiintencionalista, de manera que la carga de la prueba recae nuevamente en el bando fenomenista.

De hecho, si se analiza más detenidamente la fenomenología de los intencionales inexistentes, se debería obtener la conclusión contraria a la de los fenomenistas. Esto es, se debería concluir que la intencionalidad es una nota esencial de las experiencias cromáticas. El punto crucial de nuestra interpretación es que dichas experiencias cromáticas no intencionales tienen cierto "aire de falsedad" que las hace genuinas. Por eso, tales colores "proyectados" no son colores propiamente dichos. Su fenomenología no es cromática, en el sentido habitual; la fenomenología es diferente en ambos casos. Si padecemos una alucinación, por ejemplo, podemos determinar, atendiendo a la propia fenomenología de la experiencia, la diferencia entre estar alucinando o estar percibiendo. Y la diferencia radica en ese "querer y no poder" de los intencionales inexistentes. Los casos de alucinación o de las imágenes residuales no pueden ser verdaderos de ningún objeto. No pueden tener valor de verdad porque carecen de referencia. En el caso de las imágenes residuales, para poner otro ejemplo, si movemos nuestra mirada, ese tipo de "colores" también se mueve. Es decir, los intencionales inexistentes son ilusorios respecto de la intención, porque no determinan ninguna región espaciotemporal. Son entidades intencionales que no tienen sobre qué versar; de ahí su aire de falsedad. Así pues, puede probarse que el carácter intencional 
es el componente básico del aspecto fenoménico en el hecho de que, cuando los colores desaparecen, adquieren inmediatamente un aire de falsedad. En este sentido, la metáfora de la proyección de los fenomenistas sugiere que la intencionalidad es un segundo estadio, el estadio en el que se utilizan los qualia para representar ciertas propiedades de los objetos. En el caso de las imágenes residuales, la metáfora de la proyección funciona; en el de las alucinaciones también. Pero, en general, aplicada a las experiencias cromáticas no funciona, porque los colores no se proyectan al exterior; al menos no en el sentido anterior, según el cual la proyección es una acción extrínseca de la identidad de aquello que proyecta. De tal modo, no parece razonable suponer que el proyector sea la metáfora más conveniente para analizar las experiencias cromáticas habituales que, como decimos, son intencionales en todo momento. Cuando disminuye la eficacia referencial de las experiencias cromáticas, su identidad qua experiencias cromáticas también se debilita. La intencionalidad seguramente no es algo que se añade a los qualia cuando éstos son proyectados; es probable que sea una característica esencial de la identidad del aspecto fenoménico de las experiencias cromáticas.

Es obvio que (A) no queda justificada con esta segunda estrategia. Su justificación requiere dar un paso más: es necesario disociar la intencionalidad y la representación en el marco de las experiencias cromáticas.

\section{Distinción entre intención y representación}

La distinción asumida por los filósofos de la mente entre los qualia y los contenidos representacionales (o intencionales) se suele presentar como dicotómica. Todo contenido experiencial que no caiga en una categoría corresponde a la otra. Lo que mantenemos, sin embargo, es que no existen qualia y que la distinción entre "contenidos" sólo puede establecerse entre contenidos intencionales que a veces también son representacionales. Los estados o sucesos intencionales, como el acto perceptual de "ver los colores" adquieren también a veces, en ciertas circunstancias, contenido representacional. La justificación de esta 
distinción requiere un análisis etiológico de la percepción cromática.

El uso más frecuente de los conceptos cromáticos no es el referencial, sino el atributivo. Un concepto cromático no se usa habitualmente para señalar o identificar un color, sino para atribuir un color a un objeto. Las expresiones más corrientes en las que aparecen los conceptos cromáticos son del tipo "tal objeto es de tal color", "parece que tal objeto tiene tal color", " $x$ tiene el mismo color que $y$ ", etc. De hecho, uno de los aspectos importantes de cualquier análisis acerca de los colores es enfatizar la idea de que las experiencias cromáticas tienen un carácter presentacional o representacional. Esto es, las experiencias cromáticas no son meramente intencionales en el sentido de que se refieren, por ejemplo, a cierto aspecto fenoménico que se encuentra a alguna distancia de "aquí", que es semejante a tal otro aspecto fenoménico que se encuentra a su lado, etc. Las experiencias cromáticas presentan o representan aquello de lo que los colores son parasitarios. Si el aspecto fenoménico específico referido por el concepto 'rojo' ostensivamente definido se localiza en la superficie de un tomate, por ejemplo, lo que percibimos es que el "tomate es rojo". Y percibimos así casi todos los elementos de nuestro entorno: los plátanos maduros son amarillos, el cielo es azul, el fuego es multicolor, la nieve blanca, etcétera.

Cualquier análisis acerca del color debe elucidar la relación entre el aspecto fenoménico y el contenido presentacional de la experiencia cromática. En efecto, el color rojo definido ostensivamente forma parte de la fenomenología de la visión, pero carece de contenido presentacional; la definición ostensiva por sí misma no explica ese uso atributivo. Existe un paso no trivial de la definición ostensiva del concepto rojo (" "rojo" es esto $\rightarrow$ ") al uso atributivo de dicho concepto ("esto $\rightarrow$ es rojo"). En lo que sigue trataremos de mostrar cuál es la condición que se debe cumplir para que sea posible dar ese paso.

Intuitivamente podemos afirmar que la estabilidad fenoménica es una condición necesaria para la definición ostensiva de los colores. Ahora bien, la estabilidad fenoménica responde a la 
relativa estabilidad de los colores en relación con los objetos. Es decir, en nuestro campo visual, los objetos y los colores se correlacionan regularmente: si las condiciones de la percepción no varían, entonces el color (definido como aspecto fenoménico) del objeto tampoco varía. El aspecto fenoménico asociado a cada objeto suele ser estable (salvo excepciones tales como los objetos iridiscentes, el camaleón, el fuego, etc.) y, según trataremos de argumentar, esta estabilidad del aspecto fenoménico relativa a los objetos (a sus superficies o volúmenes) es una condición necesaria para usar los conceptos cromáticos de manera atributiva. De hecho, hay varios experimentos mentales que indican que, en algunos mundos posibles, el aspecto fenoménico no puede utilizarse de manera representacional, es decir, de manera que justifique su atribución. Estos experimentos muestran casos de mundos inestables en relación con el color. A partir de estos experimentos se concluye que, para que el aspecto fenoménico tenga contenido representacional, tal aspecto debe satisfacer el requisito de la estabilidad. En este contexto, por estabilidad entendemos lo siguiente: cuando las circunstancias del acto perceptual se mantienen estables, el aspecto fenoménico de la superficie se mantiene estable; esto es, no existen diferencias fenomenológicas entre los aspectos fenoménicos de los diferentes momentos.

Imagínese, por ejemplo, que el mundo fuera inestable en relación con el color. La característica de este mundo es que un mismo individuo, en las mismas circunstancias o en circunstancias similares, no percibe que la manzana tenga un mismo color. ${ }^{5}$ Cada cierto tiempo, la manzana cambia de color sin causa determinada. Así ocurre con todos los objetos: objetos y colores se relacionan de manera espontánea y arbitraria. En ese mundo, ¿sería posible el uso atributivo de los conceptos cromáticos definidos ostensivamente? La respuesta es que sería posible; sin embargo, esa manera de hablar no sería interesante. Esto es, la afirmación "esto $\rightarrow$ es rojo" no sería una atribución del "color" sino del "color en el instante $t$ ". Los objetos no

5 Block propone un ejemplo similar, algo más macabro: una habitación cromáticamente inestable con Eliza encerrada dentro (Block 1996, p. 21). 
tendrían un color determinado, en el sentido de que cerrados los ojos no sabríamos cuál sería el color del objeto al volver a abrirlos. No habría manera de asegurar que el color en el instante $t$ (antes de cerrar los ojos) continuaría siendo el color en el instante $t^{\prime}$ (después de cerrar los ojos). La conclusión es que en ese mundo se podría definir " "rojo" es esto $\rightarrow$ " ${ }^{6}$ pero, a diferencia del mundo en que vivimos, la afirmación "esto $\rightarrow$ es rojo" carecería de sentido. Incluso en caso de que la afirmación fuera considerada verdadera, porque se entendiera que implica el instante preciso de la enunciación, la atribución "esto $\rightarrow$ es rojo" carecería del valor práctico que en nuestro mundo hace interesantes ese tipo de afirmaciones ya que, por ejemplo, expresiones del tipo "las manzanas Red Delicious son rojas", "tráeme las manzanas rojas", "me gustan las manzanas rojas", etc., no se podrían utilizar con sentido. La única clase de objetos que podríamos discriminar mediante el concepto 'rojo' sería la de los objetos que en el instante $t$ parecen ser rojos. La clase de los "objetos rojos" sería una clase arbitrariamente determinada de la cual no se podría inferir ninguna caracterización ulterior del comportamiento cromático de los objetos. La clase de los "objetos rojos" sería similar a la clase de los "objetos que estaban nevados tal día" o a la clase de los "objetos que recibieron una patada en tal momento"; es decir, carecería de sentido afirmar que los "objetos rojos" son cromáticamente más semejantes a los "objetos anaranjados" que a los "objetos azules", porque esta afirmación dependería también del momento de la enunciación. Siempre puede fijarse un instante $t^{\prime}$ en el que los objetos azules se vean rojos — ahora según la definición ostensiva- y coincidan con los "objetos rojos" que en $t^{\prime}$ continúan siendo rojos. Por lo tanto, ese tipo de afirmación tendría un valor de

\footnotetext{
${ }^{6}$ Coincidimos con Block (1996) en que la tesis funcionalista que sostiene lo contrario depende excesivamente de la especulación empírica: "En resumen, los internistas se enfrentan a un dilema. O bien mantienen que hay diferencias funcionales innatas entre las experiencias de rojo, azul, etc., en cuyo caso dependen de la especulación empírica. O bien, por el contrario, mantienen que no habría diferencias entre las experiencias [...] de rojo, de azul, etc., en cuyo caso también dependerían de la especulación empírica" (Block 1996, p. 22).
} 
verdad variable entre clases de objetos arbitrariamente definidos, mientras que en nuestro mundo ese mismo enunciado, "los objetos rojos son cromáticamente más semejantes a los objetos anaranjados que a los objetos azules", posee un valor de verdad que comúnmente consideramos necesario, ya que da cuenta de una relación interna de los colores (todavía seguimos apelando al aspecto fenoménico de la definición ostensiva) que es atemporal; esto es, que es inherente al espacio cromático donde se estructuran los colores. Así pues, tanto la atribución cromática como la semejanza cromática correspondiente dependen, en parte, de la no inestabilidad de nuestro mundo respecto del color.

Imagínese ahora un mundo completamente transparente. Un mundo en el cual los objetos tienen color sólo en circunstancias determinadas. Así, por ejemplo, las manzanas son transparentes excepto, pongamos por caso, si se acercan a menos de diez centímetros de los ojos. A esa distancia, las manzanas parecen rojas. Cuanto más cerca de los ojos se sitúen, más rojas parecerán las manzanas. Cuando alguien se acerca la manzana a los ojos, esa persona percibe que la manzana es roja, mientras que quienes miramos la manzana desde más lejos seguiremos pensando que es transparente. Además, en ese mundo no se puede saber de qué color aparece el objeto en cuestión hasta que nos acercamos a la distancia mínima requerida. Más aún, podría haber objetos que se siguieran viendo transparentes incluso al acercarnos a esa distancia mínima, porque en ese mundo no todos los objetos tienen que parecer de algún color cuando los situamos frente a nuestros ojos. ¿En un mundo así seguiríamos atribuyendo colores a los objetos? Seguramente no. La hipótesis que consideramos más razonable es que diríamos que las manzanas son transparentes y que quien se las acerca demasiado a los ojos no puede percibir su transparencia. El color de la manzana no se consideraría una propiedad de ella, que aparece y desaparece dependiendo de la distancia que haya entre el objeto y nuestro sistema perceptual. No se diría que la manzana es roja a la distancia $d$ de los ojos. Seguramente se compararía el fenómeno de la "rojez" de la manzana con el tipo de ilusión óptica que ocurre cuando a esa distancia de los ojos un dedo se ve doble. Se 
diría que, así como parece haber dos dedos cuando, en realidad, sólo hay uno, la manzana parece "roja" cuando, en realidad, es transparente. "Rojo" sería una propiedad de la experiencia; no de la manzana. ${ }^{7}$ La experiencia cromática no sería perceptual sino introspectiva, esto es, los colores serían meras sensaciones - como el dolor - que sentimos cuando nos acercamos demasiado los objetos a los ojos abiertos. En definitiva, sostenemos que si los colores correspondiesen a los objetos de manera análoga a como lo hace el dolor, es decir, asociándose a los objetos sólo en determinadas circunstancias (el alfiler sólo pincha si acercamos suficientemente su punta a nuestra piel, el fuego sólo quema si..., etc.), entonces no utilizaríamos los conceptos cromáticos de manera atributiva. En el caso del mundo transparente, como en el anterior del mundo inestable, tampoco diríamos que "la manzana es roja" o que el "jardín es verde".

Conviene señalar, sin embargo, que este experimento mental del mundo transparente es inverso al que plantea Wittgenstein (2000 [1953], § 312). Si nosotros hemos imaginado una situación en la que los colores se correlacionan con los objetos, tal como se relaciona con ellos la sensación de dolor, Wittgenstein especula con la hipótesis inversa. ¿Cómo hablaríamos de la sensación de dolor si ésta se relacionara con los objetos con la misma regularidad que lo hacen los colores? La hipótesis de Wittgenstein parece sencilla. Al entrar en contacto con cierto tipo de superficie, esa superficie nos produce dolor. Wittgenstein concluye que si ése fuera el caso, entonces hablaríamos del dolor como ahora hablamos del color, esto es, de manera atributiva. Su argumentación es que si la aparición de la sensación de dolor junto con una clase de objetos fuera tan regular como lo es la aparición de los colores, "en ese caso deberíamos hablar de manchas de dolor en las hojas de las plantas, al igual que hablamos de manchas rojas" (2000, § 312). Según Wittgenstein, en ese caso el dolor se atribuiría al objeto, esto es, diríamos que "esa superficie es dolor". Su tesis parece ser que la regularidad es suficiente para que pueda darse un uso atributivo de los conceptos referidos

\footnotetext{
${ }^{7}$ Éste es otro argumento contra la teoría del error que considera los colores como propiedades de la experiencia.
} 
al aspecto fenoménico. Si el dolor fuera tan regular como los colores, dado que tanto el color como el dolor tienen un aspecto fenoménico que puede ser referido (exhibido) por definiciones apropiadas, entonces el uso principal del concepto 'dolor' también sería atributivo. Es decir, en lugar de atribuir el dolor a los individuos que lo padecen, el dolor se atribuiría a aquello (externo pero que también puede ser alguna parte del cuerpo) que produce esa sensación en el individuo. A nuestro juicio, ésa es una afirmación demasiado precipitada, ya que parece pasar por alto otras diferencias entre las sensaciones de dolor y el aspecto fenoménico de los colores que impiden formularla de manera consecuente. De hecho, Boghossian y Velleman afirman que no es precisamente la regularidad en la asociación con los objetos o sucesos externos lo que diferencia a los colores de la sensación de dolor (Boghossian y Velleman 1991, p. 99).

Así, la tesis que trata de defender Wittgenstein acerca de la suficiencia de la estabilidad resulta demasiado comprometida. Si aceptamos la objeción de Boghossian y Velleman al ejemplo del dolor regularmente asociado a ciertas situaciones, entonces la condición de la estabilidad no es suficiente para justificar un uso atributivo de conceptos referidos a algún tipo de aspecto fenoménico. Sin embargo, los dos experimentos mentales anteriormente mencionados sí parecen reforzar la intuición de Wittgenstein; esto es, la idea de que sin regularidad no puede darse un uso atributivo interesante de esos conceptos. La estabilidad o regularidad no parece ser una condición suficiente para justificar el uso atributivo de conceptos referidos a algún aspecto fenoménico; aunque, seguramente, sí es una condición necesaria para que se dé ese tipo de uso. Podemos concluir entonces que las experiencias cromáticas tienen contenido representacional, en el sentido de que atribuimos colores a los objetos, debido (por lo menos, en parte) a la correlación estable existente entre el aspecto fenoménico de los colores y las superficies de los objetos que caen bajo nuestro campo visual.

Este argumento, por lo tanto, permite establecer la primera distinción analítica entre el carácter cualitativo del campo visual (el aspecto fenoménico) y su contenido atributivo o representa- 
cional. A diferencia de la definición ostensiva, el propósito del uso atributivo no es caracterizar una cualidad, sino el objeto. La definición ostensiva de los conceptos cromáticos identifica (señala, nombra, exhibe,...) una cualidad del campo visual, mientras que el uso atributivo de los conceptos cromáticos presenta al objeto de una determinada manera. Y esta diferencia se transfiere también a la propia lógica de los enunciados. A diferencia de la definición " "rojo" es este $\rightarrow$ color" (cuyo significado, en ese primer estadio, era meramente fijador de referencia sin valor de verdad), las expresiones atributivas de color como "este objeto $\rightarrow$ es rojo" adquieren valor de verdad. ${ }^{8}$ De hecho, las experiencias cromáticas también son de este tipo atributivo. Desde el punto de vista psicológico, podemos afirmar incluso que no somos conscientes de estar percibiendo un color; simplemente percibimos que tal cosa es de tal color. El contenido representacional de un objeto que se ve rojo es que el objeto es rojo. El color rojo se concibe como una propiedad de los objetos que aparecen rojos en su campo visual. Desde el punto de vista del sujeto con experiencias cromáticas normales, los colores están estrechamente vinculados al soporte material del cual son parasitarios. La rojez se considera una propiedad y las experiencias cromáticas se consideran atributivas de tal propiedad (representan esa propiedad).

En resumen, lo que estos experimentos mentales muestran es la necesidad de distinguir entre las condiciones que posibilitan

\footnotetext{
${ }^{8}$ Obviamente, la expresión “'rojo” es este $\rightarrow$ color” también puede utilizarse de modo descriptivo, en cuyo caso poseería valor de verdad. La persona que está aprendiendo a utilizar los conceptos cromáticos o que tiene alterada la visión cromática puede utilizar esa expresión de manera que su enunciación sea falsa. No es la forma, sino un uso en un determinado contexto lo que confiere a esa expresión la función de fijación de la referencia (Wittgenstein 1994 [1977], II, §19). Lo mismo ocurre con la expresión "este objeto $\rightarrow$ es rojo" que tiene valor de verdad, excepto si se interpreta que el objeto en cuestión es un objeto paradigmático en cuyo caso podría entenderse que se trata también de una afirmación analítica. Se sobreentiende que en esta sección estamos considerando los usos habituales de definición ostensiva y expresión atributiva de los conceptos, tal como aparecen en nuestro discurso corriente, aunque es cierto que esas expresiones permiten otro tipo de uso sin mayor dificultad.
} 
la definición ostensiva de los colores y las condiciones que posibilitan el uso atributivo de dichos colores ostensivamente definidos. Los colores son intencionales incluso cuando carecen de contenido representacional, funcional o cognitivo. De esta manera, (A) queda justificada. Así pues, existen situaciones - por ejemplo, cuando un individuo es consciente de que desconoce en qué circunstancias se encuentra- en las cuales las experiencias cromáticas carecen de contenido representacional a pesar de que se refieren - o están dirigidas - a alguna región espaciotemporal. La estipulación (A) faculta el alejamiento tanto de los fenomenistas — que mantienen la existencia de los qualia-, como de los representacionistas - que sostienen que todo contenido experiencial es contenido representacional- $\mathrm{Y}$ es que tanto los fenomenistas como los representacionistas asumen que "representación" e "intencionalidad" se implican mutuamente: los contenidos intencionales son contenidos representacionales y viceversa. Así se explica que asuman también el siguiente condicional: si efectivamente hay algo en las experiencias cromáticas que no es reducible a su contenido representacional (o a su descripción funcional), ese algo deberían ser los qualia. Sin embargo, estas suposiciones son deudoras de la filosofía de la mente, no del análisis de la fenomenología de las experiencias cromáticas. De hecho, la existencia o no de los qualia no se fundamenta en la fenomenología de nuestras experiencias, sino en el éxito o en el fracaso de la estrategia funcionalista de reducción de la mente.

Como se ha señalado, esta estrategia es insuficiente. Ahora añadimos que además es confundente. Garantizar la existencia de los qualia esperando el fracaso funcionalista es confundente, porque la garantía de la existencia de distintos tipos de contenidos en las experiencias cromáticas no depende del fracaso de ningún programa reductivo conocido. Supóngase que la ciencia se desarrollara de manera que pudiera naturalizar todos los contenidos en una serie de enunciados científicos, sin que las experiencias cromáticas modificaran por ello sus contenidos o dejaran de tener ciertos contenidos. De ese resultado científico no se derivaría que nuestras experiencias cromáticas sólo contu- 
vieran un tipo de contenido, el representacional. El objetivo legítimo de pretender naturalizar completamente las experiencias cromáticas no debería comprometernos con una determinada visión acerca de qué tipos de contenidos contienen dichas experiencias. Y ésta es la diferencia fundamental entre este enfoque cuasifuncionalista y el que se presenta en este artículo. A nuestro juicio, es un error establecer un paralelismo entre aquello que es funcionalmente detectable y el contenido representacional. Que algo sea o no funcionalmente detectable no implica necesariamente que ese algo tenga que ser de una determinada categoría: contenido representacional, aspecto fenoménico, qualia o alguna otra.

La negación del funcionalismo no compromete necesariamente ni con el fenomenismo ni, por lo tanto, con el realismo respecto a los qualia. Lo que tratamos de defender es que el "representacionismo" y el "fenomenismo" no son las únicas opciones posibles. Podemos situarnos en una posición entre ambos que acepte el aspecto fenoménico de las experiencias cromáticas - caracterizadas como intencionales (a diferencia de los qualia) - , pero que no las considere completamente reducibles a su contenido representacional. Este enfoque, por lo tanto, es eliminativista en cuanto a los qualia (cromáticos), pero no representacionista en el sentido usual.

Como se ha expuesto, la guerra de los qualia sigue abierta porque el debate sobre la realidad de los mismos se sitúa desde los problemas que la filosofía de la mente plantea genuinamente y no desde un interés propiamente focalizado en las experiencias cromáticas. Deberíamos comenzar por preguntarnos qué tipo de entidades son las experiencias cromáticas; y no, como se suele hacer, por promover la cuestión de si el funcionalismo es una teoría completa acerca de la mente. Son dos problemas diferentes. El segundo de ellos, el de los límites del funcionalismo, sólo tiene dos respuestas posibles: sí o no. El primero, en cambio, no es tan enfático en sus soluciones. Este primer problema focaliza, sobre todo, una cuestión ontológica acerca de los estados o contenidos mentales (esto es, si una persona percibe o no un determinado contenido representacional, aspecto fenoménico o 
qualia). El segundo problema, sin embargo, formula una cuestión epistémica acerca de nuestras capacidades en determinadas circunstancias para detectar dichos estados o contenidos mentales en terceras personas. La impotencia del funcionalismo, en definitiva, no nos sitúa frente a los qualia.

\section{Conclusión}

Como se ha señalado, los análisis contemporáneos más relevantes acerca de los colores parten de una actitud reduccionista de la mente. La idea fundamental en ellos es que las experiencias cromáticas se conciben como material dispuesto para ser filtrado. Ese material admite sólo dos tipos de componentes, el que puede ser filtrado y el que no puede serlo: los contenidos representacionales y los qualia. Hemos argumentado también que ese resultado es consustancial a la estrategia del filtro porque se parte del análisis funcionalista de la mente. Evidentemente, si se parte del funcionalismo, sólo hay dos opciones posibles: la reducción completa de las experiencias cromáticas o la reducción parcial. Tanto los fenomenistas como los representacionistas han asumido que la reducción parcial implicaría la existencia de cierto tipo de entidades particulares: los qualia. A pesar de ello, ninguna de las pruebas presentadas por ambos bandos ha servido para concluir el debate sobre los qualia. Independientemente de lo interesantes y fecundos que hayan resultado los análisis de los experimentos e hipótesis cromáticos, la estrategia del filtro no sólo no ha servido para clausurar el debate; se ha convertido, además, ella misma, en una parte esencial del problema y en germen de la irresolución del debate. Porque, como hemos tratado de argumentar, ni el fracaso del funcionalismo implica los qualia ni es pertinente analizar los colores como si fueran material de filtrado.

Así, el parte de guerra presenta, a nuestro juicio, una situación bastante dramática: tanto los fenomenistas como los representacionistas deberían revisar algunos de sus supuestos. Esta necesidad se pone de manifiesto cuando consideramos que, tal y como hemos justificado por dos vías diferentes, las experiencias cromáticas son siempre intencionales a pesar de que 
sólo en ciertas circunstancias tengan contenido representacional. Si se acepta esto, se podría desarrollar una posición intermedia entre los fenomenistas y los representacionistas. Esa orientación combinaría dos ideas hasta ahora incompatibles: Por un lado, en contra de los representacionistas, mantendría que no todo contenido experiencial es contenido representacional; y, por el otro, en contra de los fenomenistas, afirmaría que las experiencias perceptuales son en todo momento intencionales - con lo cual se excluiría la posibilidad de encontrar qualia en las experiencias perceptuales- .9 Pero para llegar a esta solución debemos salir del "camino real" de la filosofía de la mente actual que, por su sesgo hacia el funcionalismo, da por supuesto que la intencionalidad de los colores depende de su contenido representacional, funcional o cognitivo.

\section{BIBLIOGRAFÍA}

Block, N., 1999 [1990], “An Excerpt from 'Troubles with Functionalism"”, en Lycan 1999, pp. 435-439.

_. 1996 [1995], "Mental Paint and Mental Latex", Philosophical Issues 7. Perception, ed. E. Villanueva, Ridgeview, Atascadero, 1996, pp. 19-50.

Boghossian, P.A. y J.D. Velleman, 1991, "Physicalist Theories of Color", Philosophical Review, vol. 100, pp. 67-106. (Reimpreso en Byrne y Hilbert 1997, pp. 105-136.)

Byrne A. y D. Hilbert (comps.), 1997, Readings on Color, MIT Press, Cambridge.

Crane, H.D. y T.P. Piantanida, 1983, “On Seeing Reddish Green and Yellowish Blue", Science, vol. 221, pp. 1078-1080.

Ezcurdia, M. y O. Hansberg (comps.), 2003, La naturaleza de la experiencia. Vol. I. Sensaciones, Instituto de Investigaciones Filosóficas-UNAM, México.

Harman, G., 1996, "Explaining Objective Color in Terms of Subjective Reactions", Philosophical Issues 7, Perception, ed. E. Villanueva, Ridgeview, Atascadero, pp. 1-18. (Reimpreso en Byrne y Hilbert 1997, pp. 247-262.)

9 De existir los qualia, esto es, las experiencias no intencionales, éstas se relacionarían con otras sensaciones, como pueden ser la nostalgia, el orgasmo, etc., cuyas referencias carecen de localización espaciotemporal genuina. 
Jackson, F., 1986, “What Mary Didn't Know”, Journal of Philosophy, vol. 83, pp. 291-295. (Versión en castellano: "Lo que María no sabía", en Ezcurdia y Hansberg 2003, pp. 111-118.)

- 1982, "Epiphenomenal Qualia", Philosophical Quarterly, vol. 32, pp. 127-136. (Versión en castellano: "Qualia epifenoménicos", en Ezcurdia y Hansberg 2003, pp. 95-110.)

Lycan, W. (comp.), 1999, Mind and Cognition, Blackwell, Oxford.

Macpherson, F., 2003, "Novel Colours and the Content of Experience", Pacific Philosophical Quarterly, vol. 84, pp. 43-66.

Nagel, T., 1974, "What Is It Like to Be a Bat?", Philosophical Review, vol. 83, pp. 435-450. (Versión en castellano: "Cómo es ser un murciélago", en Ezcurdia y Hansberg 2003, pp. 45-63.)

Shoemaker, S., 1982, "The Inverted Spectrum", Journal of Philosophy, vol. 79, pp. 357-381.

Wittgenstein, L., 2000 [1953], Philosophical Investigations, Blackwell, Oxford. (Versión en castellano: Investigaciones filosóficas, trad. Alfonso García Suárez y Ulises Moulines, Instituto de Investigaciones Filosóficas-UNAM, México, 2003 [1988].)

_, 1994 [1977], Observaciones sobre los colores, trad. Alejandro Tomasini, Paidós, Barcelona.

Recibido el 13 de febrero de 2004; aceptado el 7 de julio de 2004. 